\title{
NBQX Attenuates Excitotoxic Injury in Developing White Matter
}

\author{
Pamela L. Follett, Paul A. Rosenberg, Joseph J. Volpe, and Frances E. Jensen \\ Department of Neurology and Program in Neuroscience, Children's Hospital and Harvard Medical School, Boston, \\ Massachusetts 02115
}

\begin{abstract}
The excitatory neurotransmitter glutamate is released from axons and glia under hypoxic/ischemic conditions. In vitro, oligodendrocytes (OLs) express non-NMDA glutamate receptors (GluRs) and are susceptible to GluR-mediated excitotoxicity. We evaluated the role of GluR-mediated OL excitotoxicity in hypoxic/ ischemic white matter injury in the developing brain. Hypoxic/ ischemic white matter injury is thought to mediate periventricular leukomalacia, an age-dependent white matter lesion seen in preterm infants and a common antecedent to cerebral palsy. Hypoxia/ischemia in rat pups at postnatal day 7 (P7) produced selective white matter lesions and OL death. Furthermore, OLs in pericallosal white matter express non-NMDA GluRs at P7. Unilateral carotid ligation in combination with hypoxia $\left(6 \% \mathrm{O}_{2}\right.$ for 1
\end{abstract}

hr) resulted in selective, subcortical white matter injury with a marked ipsilateral decrease in immature and myelin basic protein-expressing OLs that was also significantly attenuated by 6-nitro-7-sulfamoylbenzo(f)quinoxaline-2,3-dione (NBQX). Intracerebral AMPA demonstrated greater susceptibility to OL injury at P7 than in younger or older pups, and this was attenuated by systemic pretreatment with the AMPA antagonist NBQX. These results indicate a parallel, maturation-dependent susceptibility of immature OLs to AMPA and hypoxia/ischemia. The protective efficacy of NBQX suggests a role for glutamate receptormediated excitotoxic OL injury in immature white matter in vivo.

Key words: white matter; glutamate receptors; $A M P A ; N B Q X$; oligodendrocytes; excitotoxicity
Differential regional vulnerability to hypoxic/ischemic brain injury may be dependent on the maturational stage of the neuronal and non-neuronal cells in a given region. A common example of such age-dependent regional susceptibility is the exclusively white matter injury seen in infants as a complication of premature birth, referred to as periventricular leukomalacia (PVL). PVL is the principal neuropathological correlate of cerebral palsy. The lesion is defined by focal necrosis of the deep periventricular white matter involving all cellular components, combined with a more diff use white matter injury that appears selective for developing oligodendrocytes (OLs) (Gilles and Averill, 1977; Dambska et al., 1989; Rorke, 1998). Reduced cerebral myelin is the most prominent subsequent cerebral abnormality observed in premature infants with evidence of PVL in the neonatal period (Paneth et al., 1990; Rorke, 1992; Iida et al., 1995; Olsen et al., 1997; Skranes et al., 1997; Inder et al., 1999).

A propensity to cerebral ischemia caused by impaired cerebrovascular autoregulation, combined with a selective vulnerability of immature OLs to ischemic injury (Volpe, 1997), may contribute to the prevalence of this lesion in the preterm infant. Developing OLs in vitro have been demonstrated to be more vulnerable than are mature, myelin basic protein (MBP)-expressing OLs to oxidative stress (Back et al., 1998) and to glutamate receptor (GluR)mediated ischemic death (Fern and Moller, 2000). OLs appear to be more vulnerable than are other glia when exposed to hypoxia/ hypoglycemia in vitro (Lyons and Kettenmann, 1998). Furthermore, a number of in vivo studies have demonstrated selective white matter injury after experimental hypoxia/ischemia in the rat brain during early postnatal development (Rice et al., 1981; Towfighi et al., 1991; Sheldon et al., 1996; Yue et al., 1997; Ikeda et al., 1998; Reddy et al., 1998; Matsuda et al., 1999).

\footnotetext{
Received July 7, 2000; revised Sept. 20, 2000; accepted Oct. 2, 2000.

This work was supported by National Institutes of Health-National Institute of Neurological Disorders and Stroke Grants NS 38475 (J.J.V. and F.E.J.), HD 18655 (J.J.V.), and NS 31718 (F.E.J.); National Institutes of Health-National Institute of Child Health and Human Development Grant HD 01359; and a grant from the Hearst Foundation (P.L.F.). We thank S. Koh and J. Fu for technical assistance and H. Kinney for discussions.

Correspondence should be addressed to Dr. Frances E. Jensen, Enders 348, Department of Neurology, Children's Hospital, 300 Longwood Avenue, Boston, MA 02115. E-mail: jensen@hub.tch.harvard.edu.

Copyright (C) 2000 Society for Neuroscience $0270-6474 / 00 / 209235-07 \$ 15.00 / 0$
}

Both clinical and experimental studies indicate that hypoxia/ ischemia is a major underlying cause of PVL. Experimental models of ischemia in immature animals implicate glutamate as a critical factor in the pathogenesis of brain injury. Hypoxic/ischemic conditions result in elevated cerebral glutamate levels in the immature rat brain, measured by in vivo microdialysis (Benveniste et al., 1984; Silverstein et al., 1991). Clinical relevance of the experimental studies is suggested by the demonstration of elevated glutamate in the CSF of term infants after perinatal hypoxia/ ischemia (Hagberg, 1992). Glutamate has been shown to be toxic to oligodendroglia in vivo and in vitro by receptor-independent (Oka et al., 1993; Yoshioka et al., 1996; Back et al., 1998) and receptormediated mechanisms (Yoshioka et al., 1995, 1996; Matute et al., 1997; McDonald et al., 1998; Pitt et al., 2000). OLs express functional GluRs in vitro, and these are exclusively of the non-NMDA subtype (Gallo et al., 1994; Patneau et al., 1994).

The purpose of this study was to examine in vivo the contribution of GluR-mediated toxicity to the selective loss of immature OLs in age-dependent cerebral white matter injury. First, we evaluated the sensitivity of immature white matter to experimental hypoxia/ ischemia at the age when the cerebral white matter of a rat is primarily populated by immature OLs. To establish whether this injury involved GluR activation, we confirmed the presence of AMPA GluRs on the vulnerable cells and then assessed the protective efficacy of treatment with the non-NMDA antagonist 6-nitro-7-sulfamoylbenzo(f)quinoxaline-2,3-dione (NBQX). We further tested the white matter selectivity and age-dependent nature of the GluR-mediated injury with glutamate agonist injections at different ages.

\section{MATERIALS AND METHODS}

Subjects. Litters of male Long-Evans rat pups (Charles River Laboratories, Wilmington, MA) were raised with dams in a temperature-controlled environment with $12 \mathrm{hr}$ light/dark cycles. All procedures were approved and in accordance with guidelines set by the institutional animal care and use committee. Pups underwent intracerebral injections or carotid ligation and hypoxia at ages postnatal day 4 (P4), P7-P8, and P10-P11. They were allowed to recover on a thermal blanket at $33-34^{\circ} \mathrm{C}$ (baseline temperature for P7-P10 rats) and returned to their dam for $48-96 \mathrm{hr}$ before being killed.

Normal development of oligodendrocytes in vivo. We evaluated changes in myelin expression with age to assess the critical ages of OL development in the neonatal rat. The corpus callosum and cortical and subcortical white matter at the level of the dorsal hippocampus were evaluated by immuno- 
cytochemistry for MBP in rats at P4, P7, P9, P11, and P18. Consistent with the results of others (Hardy and Reynolds, 1991; Back et al., 1998), our evaluation showed that MBP progressively increased during the first 3 weeks of postnatal life (see Fig. 1). No MBP staining was demonstrated at $\mathrm{P} 4 / 5(n=6)$. At P7 $(n=6)$ occasional cell bodies with limited processes expressed MBP, and these were generally present in the basal ganglia and lateral internal capsule. By P11 $(n=6)$ MBP staining is heavy throughout the corpus callosum and internal and external capsule, with MBP-positive processes extending into the overlying cortex. By P18 $(n=4)$ MBP expression was abundant in white matter tracts and throughout the cortex. Immature OLs, represented by their expression of the O1 surface marker, predominate before P11 (Gard and Pfeiffer, 1990). At P4, OL progenitors expressing $\mathrm{O} 4$ surface antigen, but not $\mathrm{O} 1$, populate the white matter. By P7 white matter is populated primarily with immature OLs expressing both $\mathrm{O} 4$ and $\mathrm{O} 1$ antigen (results not shown).

Carotid ligation with hypoxia. Selective white matter injury was produced in $\mathrm{P} 7$ rats by unilateral carotid ligation followed by hypoxia $\left(6 \% \mathrm{O}_{2}\right.$ for 1 $\mathrm{hr}$ ), modified from the method of Jensen et al. (1994). Rats were anesthetized with ether, and the proximal internal carotid artery was isolated from the sympathetic chain, clamped, and cauterized. The neck wound was closed, and the animals were allowed to recover for $1 \mathrm{hr}$ on a thermal blanket, maintaining body temperature at $33-34^{\circ} \mathrm{C}$. The rats were then placed in a sealed chamber infused with nitrogen to a level of $6 \% \mathrm{O}_{2}$, also on a thermal blanket maintaining body temperature at $33-34^{\circ} \mathrm{C}$ throughout hypoxia. Body temperature was monitored by rectal probe before and after surgery and hypoxia and did not differ between groups. After a 1-2 hr period of recovery, the rats were returned to their dam. Rats were killed 96 $\mathrm{hr}$ after injection and brains were perfused with $4 \%$ paraformaldehyde, post-fixed for $1 \mathrm{hr}$, and then cryoprotected in $30 \%$ sucrose in PBS. To determine the protective effect of GluR blockade in hypoxic/ischemic white matter injury, rats were randomized for treatment with NBQX (20 $\mathrm{mg} / \mathrm{kg} ; n=7$ ) or vehicle (sterile water; $n=9$ ) immediately after removal from hypoxia; a repeat treatment was given every $12 \mathrm{hr}$ for $48 \mathrm{hr}$.

Stereotactic intracerebral injections. To evaluate the susceptibility of immature OLs to GluR-mediated toxicity in vivo, we performed stereotactic, intracerebral injections of AMPA into the pericallosal white matter of developing rats of several ages, on the basis of a modification of the technique described by McDonald et al. (1998). Rats were anesthetized by ether inhalation, and temperature was maintained at $33-34^{\circ} \mathrm{C}$ throughout, monitored by a rectal probe. By the use of aseptic surgical technique, a scalp incision was made in the skull surface, and a burr hole was placed above the desired injection location. The rat was placed in a stereotactic apparatus, and a pulled-glass micropipette attached to a nanoinjector (Drummond Scientific) was lowered with a micromanipulator to the pericallosal white matter, $1 \mathrm{~mm}$ lateral to the midline and $1 \mathrm{~mm}$ posterior to bregma. Five nanomoles of AMPA combined with $5 \mathrm{nmol}$ of MK-801 in a $0.5 \mu \mathrm{l}$ volume were injected in the experimental groups $(\mathrm{P} 4, n=4 ; \mathrm{P} 7, n=$ 8; P11, $n=6$ ), and $5 \mathrm{nmol}$ of MK-801 alone in an equivalent volume was injected in the control group $(\mathrm{P} 7, n=8)$. Average weights of each group are as follows: P4, $8.3 \pm 0.6 \mathrm{gm}$; P7, $12.2 \pm 3.3 \mathrm{gm}$; and P11, $28.5 \pm 1.6 \mathrm{gm}$. After injection, the scalp wound was sutured closed, and the pup recovered on a heated blanket to maintain temperature at $33-34^{\circ} \mathrm{C}$ and then was returned to its dam. Rats were killed $48-72 \mathrm{hr}$ after injection and brains were perfused with $4 \%$ paraformaldehyde, post-fixed overnight, and cryoprotected in $30 \%$ sucrose solution in PBS.

To determine the effect of GluR blockade on white matter injury, rats were evaluated using systemic treatment with the AMPA antagonist NBQX $(20 \mathrm{mg} / \mathrm{kg}$, i.p.; every $12 \mathrm{hr}$ for $48 \mathrm{hr})$, with the first dose given 30 min before the intracerebral injection. A P7 litter was randomized for treatment with intraperitoneal NBQX or an equivalent volume of sterile water (vehicle).

Histological analysis and immunochemistry. Developmental studies of MBP and GluRs were performed on $50 \mu \mathrm{m}$ floating sections. For GluR analysis, sections were incubated in $10 \%$ normal goat serum in PBS to block nonspecific binding and then in $\mathrm{O} 4$ or $\mathrm{O} 1$ monoclonal antibody at 1:800 in 5\% normal goat serum. Primary antibody was labeled with fluorescein-tagged anti-mouse IgM antibody (Vector Laboratories, Burlingame, CA). For double-labeling, sections were then permeabilized with $0.1 \%$ Triton X-100 in 5\% normal goat serum in PBS, incubated overnight in anti-GluR4 (5 $\mu \mathrm{g} / \mathrm{ml}$; Chemicon, Temecula, CA), and stained with biotinylated anti-rabbit IgG followed by an avidin-Texas Red conjugate.

For all experimental rats, serial $20 \mu \mathrm{m}$ coronal sections were cut by cryostat from the anterior extent of the lateral ventricles through the posterior extent of the dorsal hippocampus. Representative sections were stained with hematoxylin and eosin (HE) for routine evaluation and with in situ end-labeling (ISEL) for detection of DNA fragmentation as a sensitive method for evidence of cell death (Wijsman et al., 1993). Mounted sections were treated with pronase $(1 \mathrm{gm} / \mathrm{ml}$; Boehringer Mannheim, Indianapolis, IN), rinsed in $2 \%$ glycine and then $\mathrm{H}_{2} \mathrm{O}$, and incubated for $1 \mathrm{hr}$ at room temperature with $50 \mu \mathrm{g} / \mathrm{ml}$ DNA polymerase I (Promega, Madison, WI) and $10 \mu \mathrm{M}$ each biotin-21-dUTP (Clontech, Cambridge, UK), dCTP, dATP, and dGTP dissolved in buffer ( $50 \mathrm{~mm}$ Tris- $\mathrm{HCl}, 5 \mathrm{~mm}$ $\mathrm{MgCl}_{2}, 10 \mathrm{~mm} \beta$-mercaptoethanol, and $\left.0.005 \% \mathrm{BSA}\right)$. Biotin end-labeled DNA fragments were detected using avidin-biotin-peroxidase complex amplification (Vectastain Elite; Vector Laboratories) with diaminobenzidine tetrahydrochloride detection.

For MBP evaluation, adjacent mounted sections were incubated in $5-10 \%$ normal goat serum for $1 \mathrm{hr}$ to block nonspecific binding and concurrently permeabilized in $0.1 \%$ Triton X-100. Slides were incubated with MBP antibody (SMA-99; Sternberger Monoclonals, Baltimore, MD) at a dilution of 1:800 in PBS with 1\% normal goat serum plus $0.1 \%$ Triton $\mathrm{X}-100$ overnight at $4^{\circ} \mathrm{C}$, followed by incubation with Oregon Green antimouse IgG antibody (Molecular Probes, Eugene, OR). For detection of immature OLs, mounted sections were blocked in $10 \%$ normal goat serum for $1 \mathrm{hr}$, incubated overnight at $4^{\circ} \mathrm{C}$ in $\mathrm{O} 1$ monoclonal antibody at a dilution of 1:800 in PBS with 5\% normal goat serum, rinsed, and incubated $1 \mathrm{hr}$ in Texas Red anti-mouse IgM antibody (Vector Laboratories).

Assessment of lesion size and statistical analysis. Data for AMPA injection experiments were collected from coronal sections stained with HE. Serial sections were evaluated for the extent of white matter and neuronal injury by comparison with the contralateral side (Towfighi et al., 1994). White matter injury was graded by a blinded observer on a $0-3$ scoring scale as follows: 0 , no discernable injury; 1 , injury limited to the immediate area of the injection site; 2 , more severe injury $<1 \mathrm{~mm}$ anterior-posterior (AP) and limited to the pericallosal region; and 3, injury extending $>1 \mathrm{~mm}$ AP and extending away from the pericallosal region into distal white matter. The mean severity at each age was compared by one-way ANOVA. The injury resulting from AMPA injection at P7 was compared against control and against NBQX-treated animals by two-tailed $t$ tests assuming unequal variances.

Coronal sections stained with HE were assessed histologically for cortical injury by light microscopy. Adjacent serial sections were also stained with OL-specific markers of immature and mature OLs and used to compare the extent of white matter depletion after hypoxia/ischemia. Three adjacent pairs of coronal sections were evaluated for each rat, at the level of the anterior hippocampus, mid-dorsal hippocampus, and posterior
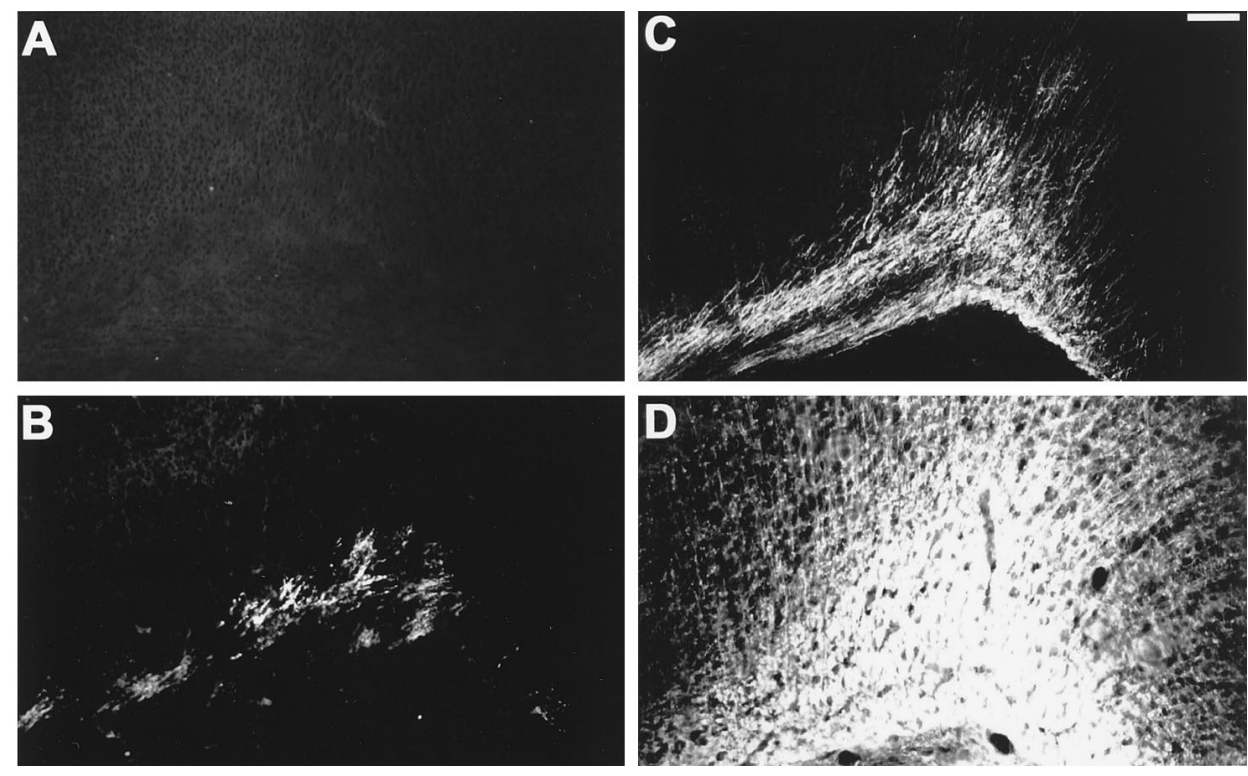

Figure 1. Developmental expression of MBPpositive cells in pericallosal white matter of immature rat brain. $A, B$, Lack of mature MBP producing OLs at $\mathrm{P} 4(A)$ and the initial appearance of sparse MBP expression at $\mathrm{P} 7(B)$. $C, D$, Rapid progression to numerous MBPproducing OLs by $\mathrm{P} 11(C)$ and a less dense but mature-appearing pattern at P18 $(D)$. Scale bar, $100 \mu \mathrm{m}$. 

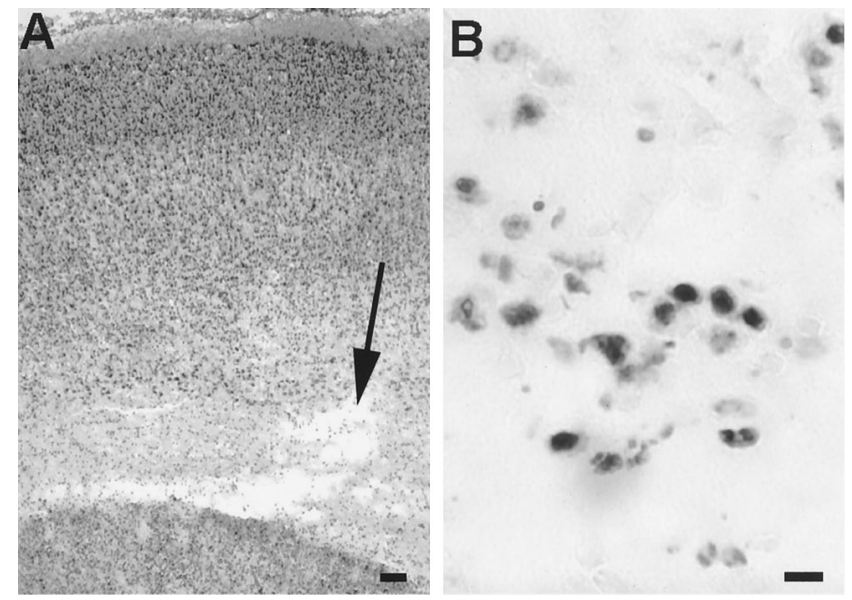

Figure 2. Selective white matter injury follows hypoxia/ischemia to immature rat brain at P7. A, Coronal section through the dorsal hemisphere of a rat killed $48 \mathrm{hr}$ after carotid ligation and hypoxia at P7, demonstrating the absence of injury to the overlying cortex. The arrow points to tissue loss in pericallosal white matter. Top of figure represents cortical surface. $B$, High-power view of dying cells in an adjacent coronal section. ISELpositive cells are common in pericallosal white matter $48 \mathrm{hr}$ after hypoxic/ ischemic insult. Scale bars: $A, 100 \mu \mathrm{m} ; B, 10 \mu \mathrm{m}$.

dorsal hippocampus, by immunocytochemistry with OL-specific antibodies for $\mathrm{O} 1$ and MBP. The white matter staining was compared ipsilateral and contralateral with the ligation, and lesion severity was assigned a value on a scale of $0-3$ as follows: 0 , the ipsilateral and contralateral hemispheres are similar; 1 , change ipsilateral to the ligation is limited to a loss of staining in the cortical processes; 2 , loss of staining includes thinning of the periventricular white matter; and 3, thinning of the white matter tracts includes a full thickness loss of staining in the capsule. A mean severity score was obtained for immature and mature OL markers in each rat. The lesion severity for each marker was compared statistically between the group treated with vehicle and the group treated with NBQX with a two-tailed $t$ test.

\section{RESULTS}

\section{Selective vulnerability of immature white matter to hypoxic ischemia}

Immunocytochemistry revealed little MBP expression before P7; a progressive increase between $\mathrm{P} 7$ and P18 indicates that white matter is predominantly populated with immature OLs at P7 (Fig. 1). Because the white matter of premature infants at high risk for hypoxic/ischemic white matter injury is also populated with immature OLs (Kinney and Back, 1998), we chose this age to deliver the hypoxic/ischemic insult. Unilateral carotid ligation followed by hypoxia $\left(6 \% \mathrm{O}_{2}\right.$ for $\left.1 \mathrm{hr}\right)$ at $\mathrm{P} 7$ resulted in a reproducible and regionally specific injury in the periventricular and subcortical
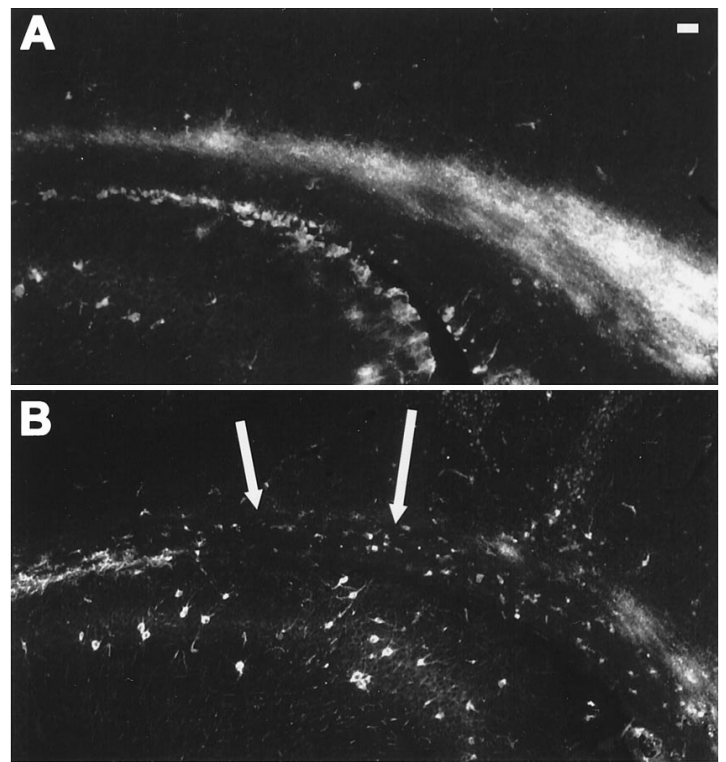

Figure 3. Loss of immature OLs in subcortical white matter after hypoxia/ ischemia. $A, B, \mathrm{O} 1$ staining of subcortical white matter tracts in coronal sections of a P11 rat. Sections are contralateral $(A)$ and ipsilateral $(B)$ to the unilateral carotid ligation that was followed by hypoxia at P7. Arrows point to an area of much reduced O1 staining after hypoxia/ischemia. Scale bar, $100 \mu \mathrm{m}$.

white matter (Fig. 2A). Injury was limited to the white matter without evidence of injury to cortical neurons. Histological observation at $48 \mathrm{hr}$ after hypoxia/ischemia demonstrated numerous ISEL positively stained cells within the subcortical white matter ipsilateral to the ligation (Fig. $2 B$ ), but not within ipsilateral overlying cortex. Immunostaining of sections from rats killed $96 \mathrm{hr}$ after hypoxia/ischemia demonstrated diminished expression of the $\mathrm{O} 1$ marker for immature OLs in seven of nine rats in subcortical white matter ipsilateral to the carotid ligation when compared with expression in the contralateral hemisphere (Fig. 3). Ipsilateral injury included the loss of MBP expression in the OL processes extending into the cortex and decreased thickness of the periventricular white matter and external capsule in four of nine rats. All rats showed an ipsilateral decrease in the presence of MBP in the OL processes extending into the cortex (Fig. 4A,B). In summary, hypoxic/ischemic injury at P7 resulted in selective white matter injury as demonstrated by the loss of MBP expression in OL processes $96 \mathrm{hr}$ later.
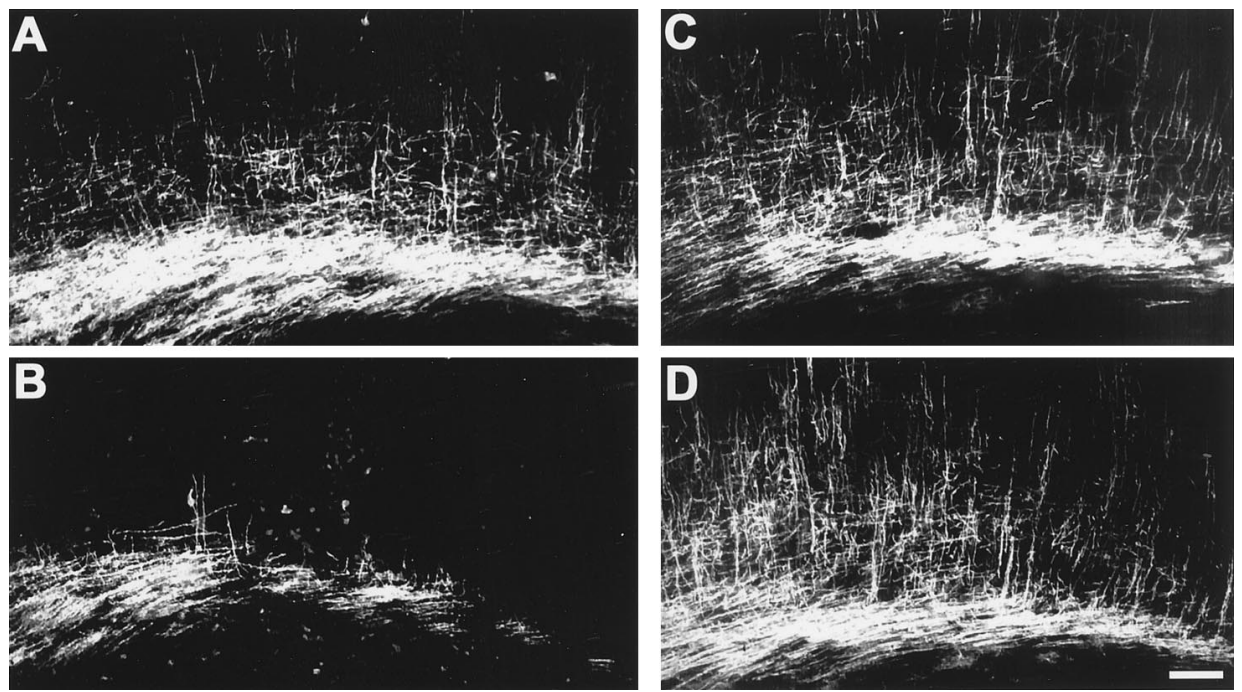

Figure 4. Effect of NBQX on MBP expression in cerebral white matter after hypoxia/ischemia. $A-D$, MBP expression in the subcortical white matter of a P11 rat after unilateral carotid ligation and hypoxia at P7, with and without NBQX treatment. MBP staining of white matter tracts contralateral $(A)$ and ipsilateral $(B)$ to the ligation in a vehicle-treated control and contralateral $(C)$ and ipsilateral $(D)$ to the ligation in a littermate post-treated with NBQX demonstrates significant attenuation of myelin loss with treatment. Scale bar, $100 \mu \mathrm{m}$. 

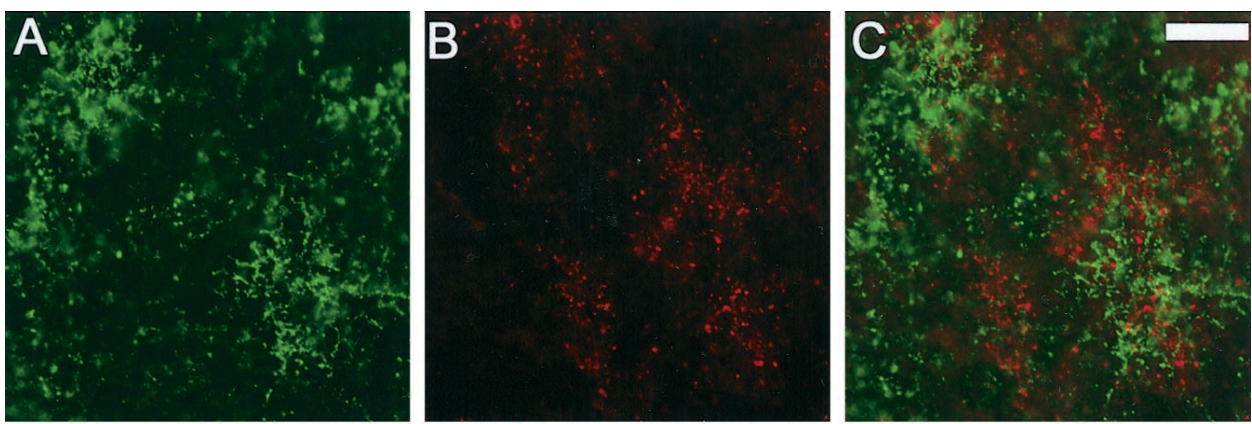

Figure 5. Immature OLs expressing O1 antibody double-label for non-NMDA GluRs in vivo at P7. D, E, O1-expressing immature OLs in the periventricular white matter of a P7 rat $(D)$ and GluR4 subunit expression in the same region $(E)$. $F$, Superimposed images demonstrating the colocalization of receptor protein with OLs at P7. Arrows point to individual $\mathrm{O} 1+$ immature OLs also expressing GluR4. $A, B$, O4-expressing OL progenitors $(A)$ and GluR4 subunit expression $(B)$ in the periventricular white matter of a P4 rat. $G, H$, MBP-expressing OLs $(G)$ and GluR4 subunit expression $(H)$ in the periventricular white matter of a P11 rat. $C, I$, Superimposed images at $\mathrm{P} 4(C)$ and P11 (I) showing the absence of colocalization at the younger and older ages. Scale bar, $50 \mu \mathrm{m}$
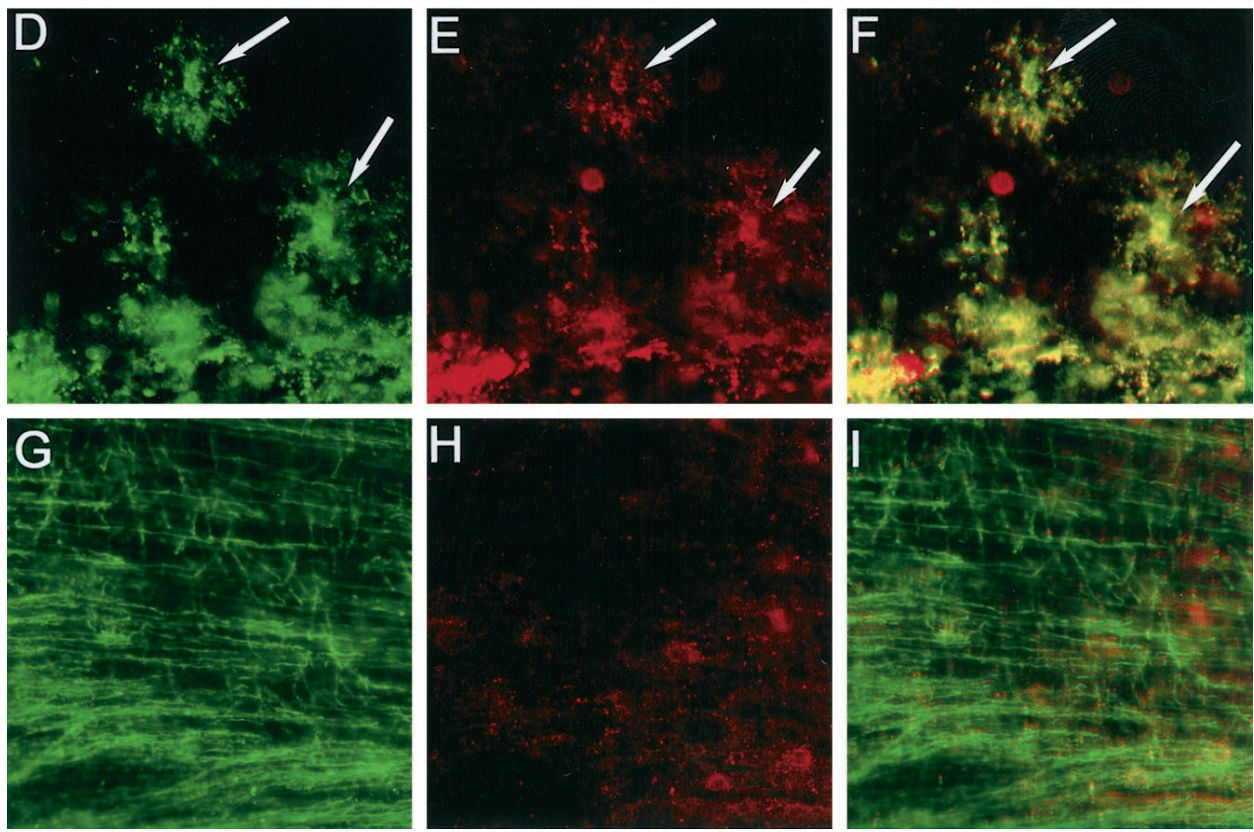

\section{Presence of AMPA-preferring GluRs in OLs at P7}

Because of the potential role of glutamate in hypoxic/ischemic white matter injury and the presence of AMPA-preferring GluRs on OLs in vitro, we evaluated whether AMPA-preferring receptors were present on immature OLs at this age in vivo. Immunocytochemical analysis using AMPA receptor subunit antibodies demonstrated robust expression of the GluR4 subunit in white matter at $\mathrm{P} 7(n=4)$. Immunocytochemistry with the $\mathrm{O} 1$ antibody to detect immature OLs (the primary OL stage present at P7) and the GluR4 antibody demonstrated widespread double-labeling in the corpus callosum, pericallosal white matter, and external and internal capsule (Fig. 5). In contrast, little coexpression of GluR4 was detected in the predominant $\mathrm{OL}$ stages seen at younger $(\mathrm{P} 4$; $\mathrm{O} 4+\mathrm{O} 1-)$ and older (P11; MBP+) ages. These data confirm the relative high expression of AMPA receptors in immature OLs in cerebral white matter at this vulnerable age.

\section{Systemic NBQX attenuates hypoxic/ischemic white matter injury}

Because of the presence of AMPA receptors on immature OLs during the time period of susceptibility to hypoxia/ischemia, we examined whether AMPA receptor blockade with NBQX would attenuate the injury. Pups treated with $\operatorname{NBQX}(n=7)$ at the termination of the period of hypoxia after carotid ligation show a marked attenuation of the ipsilateral decrease in O1 and MBP staining seen $96 \mathrm{hr}$ after the insult (Fig. 4) when compared with untreated littermate controls $(n=9)$. A semiquantitative analysis of lesion severity demonstrated significant attenuation of white matter injury in rats post-treated with $\mathrm{NBQX}$, compared with vehicle-treated controls, when evaluated for either $\mathrm{O} 1$ expression $(p<0.005)$ or MBP expression $(p<0.001)$ (Fig. 6$)$. Treated pups showed either no detectable injury (three of seven pups) or mild ipsilateral injury, generally limited to slight loss of MBP or O1 expression in the cortical processes.

\section{Systemic NBQX attenuates AMPA-mediated white matter injury}

NBQX attenuation of the selective white matter injury after hypoxia/ischemia implicates GluR-mediated toxicity as an important mechanism of injury in immature OLs. To confirm a relationship between the activation of AMPA receptors in cerebral white matter and the vulnerability to injury, we injected AMPA directly into immature white matter. Intracerebral injections of $5 \mathrm{nmol}$ of AMPA plus $5 \mathrm{nmol}$ of MK-801 produced white matter injury in P7 rat pups $(n=8)$ in the absence of significant cortical or hippocampal injury. Most P7 rats demonstrated areas of hypercellularity surrounding tissue disruption and necrosis in the pericallosal white matter, frequently with hemorrhage and with little cortical injury. Minimal to no injury was present in vehicle-injected controls (MK801 alone; $n=7)$ at the same age ( $t$ test, $p<0.001)$. The severity of white matter injury was evaluated by the size of the resulting lesion (Fig. 7). Staining with ISEL showed evidence of cell death in the white matter of the pericallosal region at P7 in rats injected with AMPA and of injury limited to the site of the needle track in controls. Systemic administration of the AMPA receptor antagonist NBQX significantly attenuated white matter injury at P7 (Fig. $7 A, t$ test, $p<0.005)$. These results suggest a receptor-mediated mechanism of injury from AMPA injections and confirm the efficacy of systemic NBQX, administered as a post-treatment.

\section{Age-dependent vulnerability of cerebral white matter to AMPA}

To determine whether AMPA toxicity was age dependent, we compared lesion size after intracerebral AMPA injections at P4, 

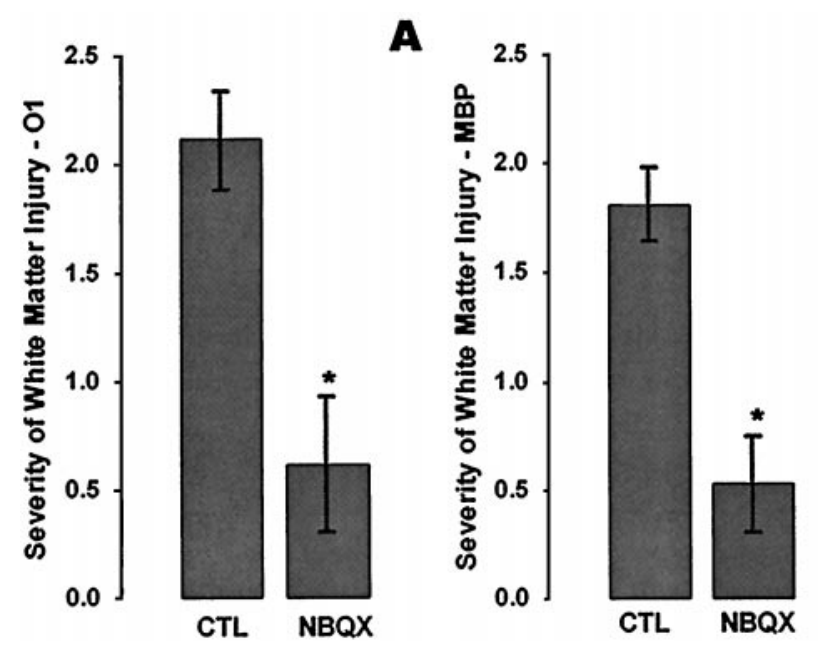

Figure 6. Evaluation of white matter injury with the OL-specific markers O1 and MBP. Comparison of the severity of white matter injury $96 \mathrm{hr}$ after hypoxia/ischemia at P7 in treated $(n=7)$ and untreated $(n=9)$ pups shows significant attenuation of injury with NBQX post-treatment $(20 \mathrm{mg} / \mathrm{kg}$, i.p.; every $12 \mathrm{hr}$ for $48 \mathrm{hr}$ ). NBQX treatment attenuates the loss of O1+ OLs $(A$ $\left.{ }^{*} p<0.005\right)$ and MBP expression $\left(B ;{ }^{*} p<0.001\right)$ ipsilateral to the ligation. $C T L$, Control.

P7, and P11. Injections at both P4 $(n=4)$ and P11 $(n=6)$ produced significantly less white matter injury than did injections at P7 (one-way ANOVA, $p<0.001$ ). Comparison of the resulting white matter injury is demonstrated by a histogram of the pericallosal lesion size (Fig. 7B). However, whereas pups injected at P4 had minimal cortical injury, animals injected at P11 exhibited widespread injury in the overlying cortex and adjacent hippocampus. These results indicate that intracerebral injections of AMPA produce an in vivo white matter lesion in an age-dependent manner; the most severe and specific lesion is at P7, and less severe and less specific lesions are at both younger (P4) and older (P11) ages.

\section{DISCUSSION}

This study suggests that the age-dependent vulnerability of OLs to hypoxic/ischemic injury may be mediated by GluR activation and may be correlated with maturational differences in GluR expression. We demonstrated an increased vulnerability of white matter to hypoxic/ischemic injury at P7, a maturational stage when white matter is populated with immature OLs. We confirmed the presence of AMPA-preferring GluR subunits on the immature OLs at this age in vivo. After hypoxia/ischemia at $\mathrm{P} 7$, systemic treatment with the AMPA receptor antagonist NBQX significantly attenuated the selective white matter injury. Furthermore, the vulnerability of white matter to intracerebral injections of AMPA appears to be age dependent, with the greatest susceptibility to injury at P7. Hypoxic/ischemic and AMPA-induced injury are each blocked by NBQX, indicating that the toxicity is receptor mediated. These results suggest that GluR-mediated toxicity is a contributing factor in the age-dependent, selective injury to developing OLs after hypoxia/ischemia in the immature brain.

The progressive development and differentiation of oligodendrocytes from progenitors to mature, myelinating oligodendrocytes has been well characterized both in vitro (Gard and Pfeiffer, 1990; Asou et al., 1995) and in vivo (LeVine and Goldman, 1988; Hardy and Reynolds, 1991, 1997). Similar developmental sequences of OL maturation are observed in the white matter of the rat and human (Kinney and Back, 1998), further supporting the use of the rat as an experimental model of PVL. Expression of MBP does not begin until P7, and this expression is followed by a rapid increase in myelin over the following few days. Therefore, this developmental stage correlates with a time in premature infants when white matter is highly vulnerable to injury.

GluR expression also appears to be maturation dependent. The presence of AMPA-preferring GluRs on OLs is well established
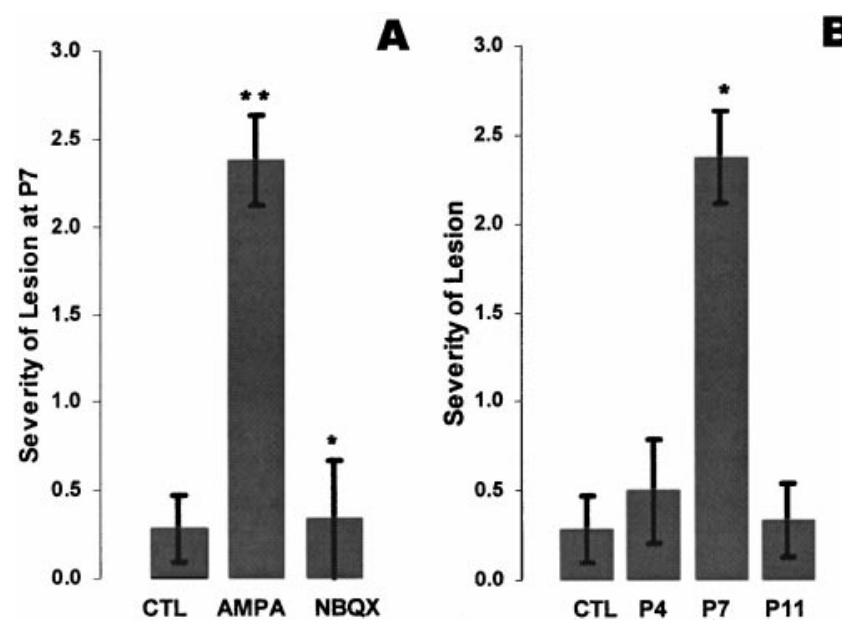

Figure 7. White matter injury after intracerebral injections of AMPA. $A$, Injury after injection with 5 mol AMPA plus $5 \mathrm{nmol}$ MK-801 $(n=8)$ was significantly more severe than with MK-801 alone (control, CTL; $n=7 ; * * p$ $<0.001) B$, The effect on rats of different ages. Significantly greater injury severity is shown in white matter at P7 $(n=8$; one-way ANOVA; $* p<$ $0.001)$ compared with that at younger $(\mathrm{P} 4 ; n=4)$ and older $(\mathrm{P} 11 ; n=6)$ ages and with MK-801 alone at P7 $(n=7)$.

(Gallo et al., 1994; Meucci et al., 1996; Matute et al., 1997), and the variable expression of AMPA receptor subtypes in different brain regions during development has been demonstrated by in situ hybridization (Pellegrini-Giampietro et al., 1991). In agreement with these results, we demonstrate relatively high levels of expression of AMPA receptors on immature OLs in vivo at $\mathrm{P} 7$, in areas vulnerable to hypoxic/ischemic injury. This age is before the time in development when expression rapidly increases in the cortex (Pellegrini-Giampietro et al., 1991; Petralia and Wenthold, 1992). The presence of GluRs on immature OLs, at an age when there is a comparative lack of expression in the cortex, may explain the relatively specific vulnerability of white matter at this maturational stage to GluR-mediated toxicity.

Moderate hypoxia/ischemia in $\mathrm{P} 7$ rats results in a selective white matter injury with relative cortical sparing. The proportion of cortical injury after hypoxia/ischemia varies with age; white matter injury is more common after cerebral hypoxia/ischemia in immature rats, whereas cortical and subcortical gray matter infarction is typically seen in the adult (Rice et al., 1981; Andine et al., 1990; Sheldon et al., 1996; Uehara et al., 1999). In addition, selective white matter injury attributable to AMPA injections is age dependent. Intracerebral AMPA caused the most selective and severe white matter injury at $\mathrm{P} 7$, with younger ages less prone to injury and older ages more susceptible to neuronal injury. White matter injury is not seen with MK-801 injections at P7, confirming that this was not a mechanical injury. Therefore, there is an agedependent injury to white matter resulting from either hypoxia/ ischemia or GluR agonist injections.

The AMPA antagonist NBQX was effective at attenuating immature white matter injury in vivo, caused either by direct receptor activation or by hypoxia/ischemia. NBQX blocked the injury at P7 caused by AMPA injections, consistent with the results of others (Yoshioka et al., 1996; McDonald et al., 1998) and suggesting a receptor-mediated cause of injury. However, in addition to blocking activation of non-NMDA receptors, NBQX has been demonstrated to induce a protective hypothermia (Young et al., 1983. Nurse and Corbett, 1996). We observed no difference in rectal temperatures between the treated and untreated groups, consistent with reports of others (Hagberg et al., 1994). A protective effect of hypothermia in $\mathrm{P} 7$ rats is significant only if present during hypoxia (Yager et al., 1993), and in the current study NBQX treatment was initiated after hypoxia. The presence of GluRs in the vulnerable cells and the attenuation of hypoxic/ischemic white matter injury 
with AMPA receptor blockade together implicate receptormediated excitotoxicity as a contributing factor in hypoxic/ischemic white matter injury in the immature brain.

The in vivo efficacy of NBQX is consistent with previous studies demonstrating attenuation of GluR-mediated excitotoxicity in vitro (Yoshioka et al., 1995, 1996; Matute et al., 1997; McDonald et al., 1998; Pitt et al., 2000). In vitro, immature OLs appear to be more susceptible to excitotoxicity than are mature OLs (Fern and Moller, 2000), and the mechanism of excitotoxicity may be calcium dependent (David et al., 1996; Yoshioka et al., 1996; Brorson et al., 1999; Li and Stys, 2000). Neither the source of elevated glutamate nor the mechanism of cell death in vivo is known; however, reverse glutamate transport from axons (Li et al., 1999; Rossi et al., 2000) or glia (Fern and Moller, 2000) has been suggested as a source. The protective efficacy of non-NMDA receptor blockade supports the hypothesis that selective injury to immature OLs by a receptormediated mechanism is sufficient to cause cerebral white matter injury in vivo.

Our results indicate that developing white matter exhibits an age window of enhanced susceptibility to GluR-mediated excitotoxicity. Injury because of hypoxia/ischemia parallels that caused by direct AMPA toxicity, with maximum selective white matter injury at P7. Cerebral white matter at this age is populated primarily by immature OLs that possess AMPA receptor subunits. In agreement with the proposed vulnerability of immature OLs to excitotoxicity by a GluR-mediated mechanism, the AMPA receptor antagonist NBQX attenuated both the AMPA-induced lesions as well as the hypoxic/ischemic white matter injury. Taken together, these results suggest that hypoxic/ischemic injury in developing white matter is mediated at least in part by excitotoxicity via glutamate receptors on immature OLs.

\section{REFERENCES}

Andine P, Thordstein M, Kjellmer I, Nordborg C, Thiringer K, Wennberg

E, Hagberg H (1990) Evaluation of brain damage in a rat model of neonatal hypoxic-ischemia. J Neurosci Methods 35:253-260.

Asou H, Hamada K, Miyazaki T, Sakota T, Hayashi K, Takeda Y, Marret S, Delpech B, Itah K, Uyemura K (1995) CNS myelination in vitro: time course and pattern of rat oligodendrocyte development. J Neurosci Res 40:519-534.

Back SA, Gan X, Rosenberg PA, Volpe JJ (1998) Maturation-dependent vulnerability of oligodendrocytes to oxidative stress-induced apoptosis caused by glutathione. J Neurosci 18:6241-6253.

Benveniste H, Drejer J, Schousboe A, Diemer NH (1984) Elevation of the extracellular concentrations of glutamate and aspartate in rat hippocampus during transient cerebral ischemia monitored by intracerebral microdialysis. J Neurochem 4:1369-1374.

Brorson JR, Zhang Z, Vandenberghe W (1999) $\mathrm{Ca}^{2+}$ permeation of AMPA receptors in cerebellar neurons expressing Glu receptor 2. J Neurosci 19:9149-9159.

Dambska M, Laure-Kamionowska M, Schmidt-Sidor B (1989) Early and late neuropathological changes in perinatal white matter damage. J Child Neurol 4:291-298.

David JC, Yamada KA, Bagwe MR, Goldberg MP (1996) AMPA receptor activation is rapidly toxic to cortical astrocytes when desensitization is blocked. J Neurosci 16:200-209.

Fern R, Moller T (2000) Rapid ischemic cell death in immature oligodendrocytes: a fatal glutamate release feedback loop. J Neurosci 20:34-42.

Gallo V, Patneau DK, Mayer ML, Vaccarino FM (1994) Excitatory amino acid receptors in glial progenitor cells: molecular and functional properties. Glia 11:94-101.

Gard AL, Pfeiffer SE (1990) Two proliferative stages of the oligodendrocyte lineage (A2B5+O4- and $\mathrm{O} 4+\mathrm{GalC}-$ ) under different mitogenic control. Neuron 5:615-625.

Gilles FH, Averill DR (1977) Neonatal endotoxin encephalopathy. Ann Neurol 2:49-56.

Hagberg H (1992) Hypoxic-ischemic damage in the neonatal brain: excitatory amino acids. Dev Pharmacol Ther 18:139-144.

Hagberg H, Gilland E, Diemer N, Andine P (1994) Hypoxia-ischemia in the neonatal rat brain: histopathology after post-treatment with NMDA and non-NMDA receptor antagonists. Biol Neonate 66:205-213.

Hardy R, Reynolds R (1991) Proliferation and differentiation potential of rat forebrain oligodendroglial progenitors both in vitro and in vivo. Development 111:1061-1080.

Hardy R, Reynolds R (1997) Oligodendroglial progenitors labeled with the $\mathrm{O} 4$ antibody persist in the adult rat cerebral cortex in vivo. J Neurosc Res 47:455-470.

Iida K, Takashima S, Ueda K (1995) Immunohistochemical study of my- elination and oligodendrocyte in infants with periventricular leukomalacia. Pediatr Neurol 13:296-304.

Ikeda T, Murata Y, Quilligan EJ, Choi BH, Parer JT, Doi S, Park SD (1998) Physiologic and histologic changes in near-term fetal lambs. Am J Obstet Gynecol 178:24-32.

Inder TE, Huppi PS, Warfield S, Kikinis R, Zientara GP, Barns PD, Jolesz F, Volpe JJ (1999) Periventricular white matter injury in the premature infant is followed by reduced cerebral cortical gray matter volume at term. Ann Neurol 46:755-760.

Jensen FE, Gardner G, Williams A, Gallop P, Aizenman E, Rosenberg PA (1994) The putative essential nutrient pyrroloquinoline quinone is neuroprotective in a rodent model of hypoxic/ischemic brain injury. Neuroscience 62:399-406.

Kinney HC, Back SA (1998) Human oligodendroglial development: relationship to periventricular leukomalacia. Semin Pediatr Neurol 5:180-189.

LeVine SM, Goldman JE (1988) Spatial and temporal patterns of oligodendrocyte differentiation in rat cerebrum and cerebellum. J Comp Neurol 277:441-455.

Li S, Stys PK (2000) Mechanisms of ionotropic glutamate receptormediated excitotoxicity in isolated spinal cord white matter. J Neurosci 20:1190-1198.

Li S, Mealing GAR, Morley P, Stys PK (1999) Novel injury mechanism in anoxia and trauma of spinal cord white matter: glutamate release via reverse $\mathrm{Na}+$-dependent glutamate transport. J Neurosci 19:RC16.

Lyons SA, Kettenmann H (1998) Oligodendrocytes and microglia are selectively vulnerable to combined hypoxia and hypoglycemia injury in vitro. J Cereb Blood Flow Metab 18:521-530.

Matsuda T, Okuyama K, Cho K, Hoshi N, Matsumoto Y, Kobayashi Y, Fujimoto S (1999) Induction of antenatal periventricular leukomalacia by hemorrhagic hypotension in the chronically instrumented fetal sheep. Am J Obstet Gynecol 181:725-730.

Matute C, Sanchez-Gomez MV, Martinez-Millan L, Miledi R (1997) Glutamate receptor-mediated toxicity in optic nerve oligodendrocytes. Proc Natl Acad Sci USA 94:8830-8835.

McDonald JW, Althomsons SP, Krzysztof LH, Choi DW, Goldberg MP (1998) Oligodendrocytes from forebrain are highly vulnerable to AMPA/kainate receptor-mediated excitotoxicity. Nat Med 4:291-297.

Meucci O, Fatatis A, Holzwarth JA, Miller RJ (1996) Developmental regulation of the toxin sensitivity of $\mathrm{Ca}^{2+}$-permeable AMPA receptors in cortical glia. J Neurosci 16:519-530.

Nurse S, Corbett D (1996) Neuroprotection after several days of mild, drug-induced hypothermia. J Cereb Blood Flow Metab 16:474-480.

Oka A, Belliveau MJ, Rosenberg A, Volpe JJ (1993) Vulnerability of oligodendroglia to glutamate: pharmacology, mechanisms, and prevention. J Neurosci 13:1441-1453.

Olsen P, Paakko E, Vainionpaa L, Pyhtinen J, Jarvelin MR (1997) Magnetic resonance imaging of periventricular leukomalacia and its clinical correlation in children. Ann Neurol 41:754-761.

Paneth N, Rudelli R, Monte W, Rodriguez E (1990) White matter necrosis in very low birth weight infants: neuropathologic and ultrasonographic findings in infants surviving six days or longer. J Pediatr 116:975-984.

Patneau DK, Wright PW, Wisden W (1994) Glial cells of the oligodendrocyte lineage express both kainate- and AMPA-preferring subtypes of glutamate receptor. Neuron 12:357-371.

Pellegrini-Giampietro DE, Bennet MVL, Zukin RS (1991) Differential expression of three glutamate receptor genes in developing rat brain: an in situ hybridization study. Proc Natl Acad Sci USA 88:4157-4161.

Petralia RS, Wenthold RJ (1992) Light and electron immunocytochemical localization of AMPA-selective glutamate receptors in the rat brain. J Comp Neurol 318:329-354.

Pitt D, Werner P, Raine CS (2000) Glutamate excitotoxicity in a model of multiple sclerosis. Nat Med 6:67-70.

Reddy K, Mallard C, Marks K, Bennet L, Gunning M, Gunn A, Gluckman P, Williams C (1998) Maturational change in the cortical response to hypoperfusion injury in the fetal sheep. Pediatr Res 43:674-682.

Rice JEI, Vannucci RC, Brierley JB (1981) The influence of immaturity on hypoxic-ischemic brain damage in the rat. Ann Neurol 9:131-141.

Rorke LB (1992) Anatomical features of the developing brain implicated in pathogenesis of hypoxic-ischemic injury. Brain Pathol 2:211-221.

Rorke LB (1998) Pathology of perinatal brain injury. New York: Raven.

Rossi DJ, Oshima T, Attwell D (2000) Glutamate release in severe brain ischaemia is mainly by reversed uptake. Nature 403:316-321.

Sheldon RA, Chuai J, Ferriero DM (1996) A rat model for hypoxicischemic brain damage in very premature infants. Biol Neonate 69:327-341.

Silverstein FS, Naik B, Simpson J (1991) Hypoxia-ischemia stimulates hippocampal glutamate efflux in perinatal rat brain: an in vivo microdialysis study. Pediatr Res 30:587-590.

Skranes JS, Vik T, Nilsen G, Smevik O, Andersson HW, Brubakk AM (1997) Cerebral magnetic resonance imaging and mental and motor function of very low birth weight children at six years of age. Neuropediatrics 28:149-154.

Towfighi J, Yager JY, Housman C, Vannucci RC (1991) Neuropathology of remote hypoxic-ischemic damage in the immature rat. Acta Neuropathol (Berl) 81:578-587.

Towfighi J, Housman C, Vannucci RC, Heitjan DF (1994) Effect of uni- 
lateral perinatal hypoxic-ischemic brain damage on the gross development of opposite cerebral hemisphere. Biol Neonate 65:108-118.

Uehara H, Yoshioka H, Kawase S, Nagai H, Ohmae T, Hasegawa K, Sawada T (1999) A new model of white matter injury in neonatal rats with bilateral carotid artery occlusion. Brain Res 837:213-220.

Volpe JJ (1997) Brain injury in the premature infant: from pathogenesis to prevention. Brain Dev 19:519-534.

Wijsman JH, Jonker RR, Keijzer R, van de Velde CJ, Cornelisse CJ, van Dierendonck JH (1993) A new method to detect apoptosis in paraffin section: in situ end-labeling of fragmented DNA. J Histochem Cytochem 41:7-12.

Yager J, Towfighi J, Vannucci RC (1993) Influence of mild hypothermia on hypoxic-ischemic brain damage in the immature rat. Pediatr Res 34:525-529.
Yoshioka A, Hardy M, Younkin DP, Grinspan JB, Stern JL, Pleasure D (1995) $\alpha$-Amino-3-hydroxy-5-methyl-4-isoxazoleproprionate (AMPA) receptors mediate excitotoxicity in the oligodendroglial lineage. J Neurochem 64:2442-2448.

Yoshioka A, Bacskai B, Pleasure D (1996) Pathophysiology of oligodendroglial excitotoxicity. J Neurosci Res 46:427-438.

Young RS, Olenginski TP, Yager J, Towfighi J (1983) The effect of graded hypothermia on hypoxic-ischemic brain damage: a neuropathologic study in the neonatal rat. Stroke 14:929-934.

Yue X, Mehmet H, Penrice J, Cooper C, Cady E, Wyatt JS, Reynolds EO, Edwards AD, Squier MV (1997) Apoptosis and necrosis in the newborn piglet brain following transient cerebral hypoxia-ischemia. Neuropathol Appl Neurobiol 23:16-25. 